\title{
Cost-effectiveness of increasing vaccination in high-risk adults aged 18-64 Years: a model-based decision analysis
}

\author{
Angela R. Wateska', Mary Patricia Nowalk², Richard K. Zimmerman², Kenneth J. Smith ${ }^{1 *}$ (D) and Chyongchiou J. Lin ${ }^{2}$
}

\begin{abstract}
Background: Adults aged 18-64 years with comorbid conditions are at high risk for complications of certain vaccine-preventable diseases, including influenza and pneumococcal disease. The 4 Pillars ${ }^{\text {TM }}$ Practice Transformation Program (4 Pillars Program) increases uptake of pneumococcal polysaccharide vaccine, influenza vaccine and tetanus-diphtheria-acellular pertussis vaccine by 5-10\% among adults with high-risk medical conditions, but its cost-effectiveness is unknown.
\end{abstract}

Methods: A decision tree model estimated the cost-effectiveness of implementing the 4 Pillars Program in primary care practices compared to no program for a population of adults 18-64 years of age at high risk of illness complications over a 10 year time horizon. Vaccination rates and intervention costs were derived from a randomized controlled cluster trial in diverse practices in 2 U.S. cities. One-way and probabilistic sensitivity analyses were conducted.

Results: From a third-party payer perspective, which considers direct medical costs, the 4 Pillars Program cost $\$ 28,301$ per quality-adjusted life year gained; from a societal perspective, which adds direct nonmedical and indirect costs, the program was cost saving and more effective than no intervention. Cost effectiveness results favoring the program were robust in sensitivity analyses. From a public health standpoint, the model predicted that the intervention reduced influenza cases by 1.4\%, with smaller decreases in pertussis and pneumococcal disease cases.

Conclusion: The 4 Pillars Practice Transformation Program is an economically reasonable, and perhaps cost saving, strategy for protecting the health of adults aged $<65$ years with high-risk medical conditions

Keywords: High-risk adults, Pneumococcal vaccine, Influenza vaccine, Tdap vaccine, Primary care, Adult vaccination

\section{Background}

Adults aged 18-64 years with immunocompromising and other chronic medical conditions are becoming a sizable proportion of the U.S. population; of U.S. adults aged $50-64$ years, $30.6 \%$ had a least one of these conditions [1]. These individuals are at high risk for complications of certain vaccine-preventable diseases, including influenza and pneumococcal disease [2]. Yet, vaccination rates among all adults aged 19-64 years are low overall, with influenza vaccination coverage from $31.5-47.7 \%$ and tetanus vaccination $62.6-64.7 \%$ among all $19-64$ year

\footnotetext{
*Correspondence: smithkj2@upmc.edu

'Department of Medicine, University of Pittsburgh School of Medicine, 200

Meyran Ave., Suite 200, Pittsburgh, PA 15213, USA

Full list of author information is available at the end of the article
}

olds, and pneumococcal vaccination in only $20.3 \%$ of high-risk individuals in this age group [3]. Few studies have examined attempts to overcome barriers to vaccine uptake in 19-64 year olds.

A recent exception is the 4 Pillars $^{\mathrm{Tw}}$ Practice Transformation Program (4 Pillars Program) which has increased vaccination rates among varied populations in diverse medical practices [4-8]. The program, developed through CDC support and owned and licensed by the University of Pittsburgh, consists of medical practicebased tools to improve: 1) the convenience of vaccination services, 2) communication with patients about the importance of immunization and the availability of vaccines, 3) office systems to facilitate immunization, and 4) motivation through an office immunization champion 
who monitors progress and encourages adherence to vaccination-promoting office practices. Although the 4 Pillars Program (4pillarstoolkit.pitt.edu) requires a commitment to long-term practice systems changes, it has been shown to be cost-effective among adults aged 65 years and older [9], but its cost-effectiveness in highrisk non-elderly adults is unknown.

Using changes in vaccination rates as a result of the 4 Pillars Program, where trial-based vaccination rates in high-risk adults aged 18-64 improved by $5-10 \%$, and cost estimates for implementing the program, we examined the cost-effectiveness of the 4 Pillars Program for improving vaccination rates among adults $18-64$ years of age with immunocompromising and other chronic medical conditions that confer a high risk of vaccinepreventable disease. This analysis was motivated, in part, by the modest program-related improvement in vaccination rates carrying with it a relatively high burden of investment.

\section{Methods}

Using a decision tree model (Fig. 1), constructed in TreeAge Pro 2017 (TreeAge Software, Williamstown MA), the cost-effectiveness of implementing the 4 Pillars Program in primary care practices was compared to no program in identical hypothetical cohorts of high-risk adults 18-64 years of age. High-risk adults were defined, using CDC definitions, as those with one or more comorbid or immunocompromising conditions, with comorbid conditions including one or more of the following: chronic heart, lung, or liver diseases, alcoholism, diabetes mellitus, or individuals who smoke cigarettes; immunocompromising conditions were HIV disease, hematologic malignancies, dialysis, nephrotic syndrome, organ or bone marrow transplant, sickle cell disease, immune deficiency, or current immunosuppressive therapy. Pneumococcal polysaccharide vaccine (PPSV), influenza vaccine, and tetanus-diphtheriaacellular pertussis (Tdap) vaccination rates and intervention costs from a randomized controlled cluster trial implementing the 4 Pillars program in two U.S. cities were used. The cohort of high-risk 18-64 year-olds not receiving the intervention was assigned baseline vaccination rates for high-risk 18-64 year olds from the trial, while an identical cohort receiving the intervention had absolute increases in vaccination uptake rates for this age and risk group from the end of the two-year trial. Ranges of vaccine coverage in both intervention and non-intervention cohorts came from uptake rates in different trial sites. Because our unit of analysis was the cohort and overall vaccine protection within the cohort, we assumed that the probability of receiving one vaccine was independent of receiving the others and, based on these probabilities, portions of the cohort could potentially receive one, two, or three vaccines, or none at all. Despite knowing that persons receiving one vaccine are more likely to receive another, we assumed this independence because there is no cross protection from one vaccine to another. Thus, protection afforded by any one vaccine is solely determined by the proportion of the population receiving that vaccine. Based on current guidelines regarding pneumococcal immunization, we assumed that immunocompromised persons receive both PPSV and the 13-valent pneumococcal conjugate vaccine (PCV13), while the remaining high-risk persons received PPSV only.

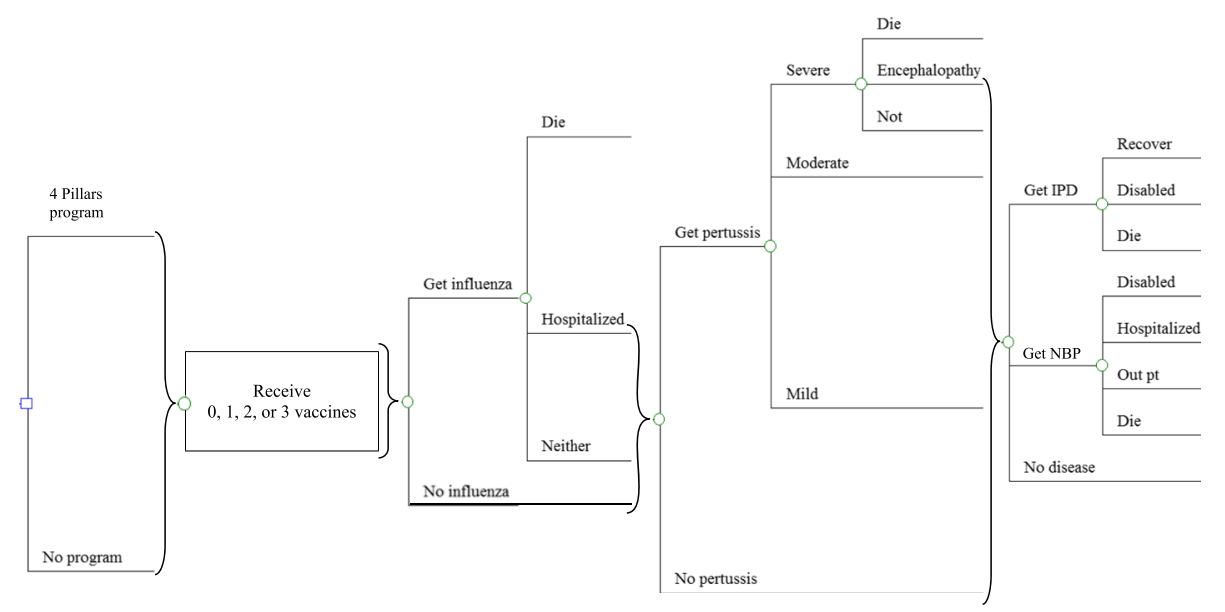

Fig. 1 Decision Tree diagram. At the square decision node, identical hypothetical cohorts of high-risk 18-64 year olds could receive the implementation program or not. Nodes to the right of brackets are connected to all branches to the left of brackets. At each circular chance node, potions of cohorts could receive vaccines, become ill with influenza, pertussis, and/or pneumococcal disease, with or without complications, based on the 10-year probability of those events. Disease probabilities were based on vaccines received and vaccine effectiveness. IPD = invasive pneumococcal disease; $\mathrm{NBP}=$ non-bacteremic pneumococcal pneumonia 
Table 1 lists model parameter base case values and their corresponding ranges examined in sensitivity analyses. Each parameter was varied individually in 1-way sensitivity analysis over the ranges shown in Table 1. All parameters were also simultaneously varied over their distributions 5000 times in a probabilistic sensitivity analysis, with results depicted in Fig. 2 as acceptability curves. As the original study did not collect illness rate data, illness attack rates for unvaccinated high-risk individuals 18-64 years of age were derived from the medical literature; whereas illness risk for vaccinated individuals was calculated as each illness's attack rate in the unvaccinated multiplied by 1 minus vaccine effectiveness. Age-specific estimates of vaccine effectiveness for influenza and Tdap were obtained from the medical literature. For pneumococcal vaccine, age- and comorbidity-specific Delphi expert panel estimates [10] of yearly effectiveness - PPSV against invasive pneumococcal disease (IPD), PCV13 against IPD, and PCV13 against non-bacteremic pneumococcal pneumonia (NBP) - were each averaged over a 10-year period, with overall effectiveness calculated as illness-specific vaccine effectiveness multiplied by the vaccine-specific pneumococcal illness serotype prevalence to calculate illnessspecific vaccine effectiveness against all serotypes, adjusted for recent pneumococcal vaccine trial results [11]. In the analysis, we assume that PPSV is ineffective against NBP, based on Delphi panel estimates [10], which could bias against interventions to improve vaccination rates. Over the model 10-year time horizon, program-related improvement in vaccination rates was assumed to be constant. However, these improvements were varied widely in sensitivity analyses to test this assumption, and to determine what levels of improvement would be necessary for the program to be favored.

In the decision analysis model (Fig. 1), when considering public health outcomes, influenza outcomes included illness requiring no treatment, outpatient treatment, hospitalization, or death, based on probabilities from the literature [12]. Outcomes for pneumococcal disease included IPD and NBP, with risks of disability and mortality for each. NBP cases could either be treated as outpatient or inpatient, while all IPD cases were assumed to be hospitalized [13]. Individuals could have mild, moderate, or severe pertussis; with severe pertussis, there were additional risks of mortality or encephalopathy $[14,15]$. Diphtheria and tetanus outcomes were not included in the analysis due to rarity of those diseases. Model outputs for vaccine-preventable illness, hospitalization, and death were included as proportions of the cohort, which are presented as percentages and as likelihoods per 100,000 in the cohort.

In the cost effectiveness analysis, vaccine-preventable illnesses occurring over the 10-year model time horizon had effectiveness modeled as lifetime per-person losses in quality adjusted life years (QALY) due to those illnesses; disability and death resulted in QALY losses based on the discounted life expectancy of the cohort. Costs of the 4 Pillars Program were calculated based on questionnaire data completed by study sites on personnel time and materials costs devoted to the program during its implementation and maintenance phases, which totaled $\$ 1.78$ per eligible patient. These costs, which included estimates of time required for all components of the 4 Pillars, including physician immunization champions, were varied over ranges based on differences in observed site-specific costs and on alternative costing assumptions. These costs varied from $\$ 1700-\$ 5400$ per center per year, and are further enumerated in Additional file 1: Table S1. All other costs and quality of life utilities were obtained from the medical literature and U.S. databases [11, 12, 14, 16-18]. All costs were inflated as necessary to 2015 U.S. dollars using the Consumer Price Index. In accordance with recently updated recommendations for cost-effectiveness analysis [19], our results include both third party payer and societal perspectives. Third party payer perspective costs include direct medical costs from illness outcomes, vaccinations and intervention program, and cost of death; whereas societal perspective costs additionally included the costs of lost productivity from illness, disability, and death. Cost-effectiveness results were calculated by comparing each strategy's per person total costs for the intervention, vaccination, and illness and effectiveness in quality adjusted life years to determine the incremental cost and incremental effectiveness between strategies. Dividing incremental cost by incremental effectiveness calculates the incremental cost effectiveness ratio (ICER), producing the incremental cost per quality adjusted life year gained for one strategy compared to the other.

\section{Results}

The model demonstrated substantial public health benefits of the intervention over a 10-year period for adults 18-64 years with high-risk medical conditions (Table 2). Specifically, the intervention program reduced influenza cases by $1.37 \%$ and influenza hospitalizations by $0.028 \%$. With the intervention program, the model also predicted smaller but substantial decreases in pertussis and in pneumococcal disease.

Table 3 outlines cost-effectiveness analysis results from the model. From a third party payer perspective, considering only direct medical costs, the 4 Pillars Program per-person total vaccination and illness costs were \$1642, \$17.88 greater than no intervention, while losing 0.00063 QALYs compared with no program, thus the program had an ICER of $\$ 28,301$ per QALY gained. 
Table 1 Model parameter values for high-risk adults aged 18-64 years

\begin{tabular}{|c|c|c|c|}
\hline Parameter & Base case & Range & Source \\
\hline Probabilities & $\%$ & $\%$ & \\
\hline \multicolumn{4}{|l|}{ Vaccination probability with no program } \\
\hline Influenza & 52.1 & $26.4-85.7$ & 4 Pillars $^{\mathrm{TM}}$ \\
\hline Tdap & 37.9 & $4.2-85.7$ & 4 Pillars $^{\top M}$ \\
\hline Pneumococcal vaccines & 43.4 & $16.7-61.9$ & 4 Pillars $^{T M}$ \\
\hline \multicolumn{4}{|l|}{ Absolute increase in vaccine uptake with program } \\
\hline Influenza & 4.7 & $0-15.2$ & 4 Pillars $^{\mathrm{TM}}$ \\
\hline Tdap & 11.5 & $0-27.3$ & 4 Pillars $^{T M}$ \\
\hline Pneumococcal vaccines & 12.3 & $4.1-28.6$ & 4 Pillars $^{\mathrm{TM}}$ \\
\hline \multicolumn{4}{|l|}{ Vaccine effectiveness } \\
\hline Influenza & 59.0 & $20-67$ & [28] \\
\hline Tdap (10 year average) & 24.5 & $0-95$ & [29] \\
\hline Pneumococcal vaccines ( 10 year average ${ }^{a}$ ) & & & Calculated $[10,11]$ \\
\hline $\begin{array}{l}\text { PPSV alone (pts with comorbid conditions) } \\
\text { Against IPD }\end{array}$ & 46.5 & $22-72$ & \\
\hline Against NBP & 0 & - & \\
\hline $\begin{array}{l}\text { PPSV and PCV13 (immunocompromised pts) } \\
\text { Against IPD }\end{array}$ & 36.3 & $19-56$ & \\
\hline Against NBP & 25.8 & $14-40$ & \\
\hline \multicolumn{4}{|l|}{ Pneumococcal illness serotype prevalence } \\
\hline PCV13 serotypes & 30.7 & $6.8-63$ & [16] \\
\hline PPSV serotypes & 67.6 & $51-82$ & [16] \\
\hline Relative likelihood of immunocompromised given high-risk & $10.7 \%$ & $5-15 \%$ & [1] \\
\hline \multicolumn{4}{|l|}{ Probability of illness without vaccinations (yearly) } \\
\hline Influenza & 6.6 & $3.2-10$ & [12] \\
\hline Pertussis & 0.202 & $0.101-0.303$ & [15] \\
\hline IPD (pts with comorbid conditions) & 0.012 & $0.006-0.018$ & [13] \\
\hline IPD (immunocompromised pts) & 0.074 & $0.037-0.111$ & [13] \\
\hline NBP (pts with comorbid conditions) & 1.44 & $0.72-2.16$ & [13] \\
\hline NBP (immunocompromised pts) & 9.05 & $4.5-13.58$ & [13] \\
\hline Relative likelihood of outpatient treatment (vs. inpatient) & 90.07 & $76-98$ & [13] \\
\hline IPD disability & 6.02 & $4-8$ & [13] \\
\hline IPD mortality & 15.9 & $13.8-35.2$ & [13] \\
\hline NBP disability & 3 & $2-4$ & Estimate \\
\hline NBP mortality & 6.3 & $5.3-14.3$ & [13] \\
\hline Case-hospitalization, influenza & 1.93 & $0.65-3.21$ & [12] \\
\hline Case-mortality, influenza & 0.134 & $0.04-.224$ & [12] \\
\hline Outpatient influenza & 62.5 & $38.9-86.1$ & [12] \\
\hline \multicolumn{4}{|l|}{ Pertussis severity relative likelihood } \\
\hline Mild & 11 & $5-17$ & [14] \\
\hline Relative likelihood of treatment (vs. no treatment) & 37.2 & $20-55$ & [14] \\
\hline Moderate & 86 & $75-90$ & [14] \\
\hline Severe (hospitalized) & 3 & $0-6$ & [14] \\
\hline Encephalopathy, given severe & 1.43 & $0-3$ & [14] \\
\hline Mortality, given severe & 0.86 & $0-2$ & [14] \\
\hline
\end{tabular}


Table 1 Model parameter values for high-risk adults aged 18-64 years (Continued)

\begin{tabular}{|c|c|c|c|}
\hline Parameter & Base case & Range & Source \\
\hline Costs (base year 2015) & US\$ & US\$ & \\
\hline \multicolumn{4}{|l|}{ Vaccines } \\
\hline Influenza & 10.69 & $6.64-32.75$ & {$[30]$} \\
\hline Tdap & 37.55 & $20.18-42.61$ & {$[30]$} \\
\hline PPSV & 78.90 & $26.60-130$ & {$[30]$} \\
\hline PCV13 & 159.60 & $96.1-220$ & {$[30]$} \\
\hline Vaccine administration, per vaccine & 25.08 & $20-30$ & [31] \\
\hline Implementation program, per eligible person & 1.78 & $0.70-2.26$ & 4 Pillars $^{\mathrm{TM}}$ \\
\hline \multicolumn{4}{|l|}{ Mild pertussis, when treated } \\
\hline Third-party payer perspective & 305 & $153-457$ & [17] \\
\hline Societal perspective & 882 & $441-1323$ & [17] \\
\hline \multicolumn{4}{|l|}{ Moderate pertussis } \\
\hline Third-party payer perspective & 424 & $212-636$ & [17] \\
\hline Societal perspective & 1001 & $501-1502$ & [17] \\
\hline \multicolumn{4}{|l|}{ Severe pertussis } \\
\hline Third-party payer perspective & 7850 & $3925-11,775$ & [14] \\
\hline Societal perspective & 8261 & $4130-12,391$ & [14] \\
\hline Influenza (outpatient) & 944 & $472-1416$ & [12] \\
\hline Hospitalized influenza & 53,212 & $26,606-79,818$ & {$[12]$} \\
\hline \multicolumn{4}{|l|}{ Pneumococcal disease } \\
\hline Invasive pneumococcal disease & 30,745 & $15,373-46,118$ & [13] \\
\hline Non-bacteremic pneumococcal pneumonia (hospitalized) & 17,466 & $8733-26,199$ & {$[13]$} \\
\hline Non-bacteremic pneumococcal pneumonia (outpatient) & 571 & $286-857$ & [13] \\
\hline Disability & 32,987 & $16,494-49,481$ & [13] \\
\hline Cost of death & 153,085 & $76,543-229,628$ & {$[12]$} \\
\hline Cost of lost productivity & 671,226 & $335,613-1,006,839$ & [12] \\
\hline Cost of lost day of productivity & 187 & $158-223$ & {$[12]$} \\
\hline \multicolumn{4}{|l|}{ Utilities } \\
\hline \multicolumn{4}{|l|}{ Influenza } \\
\hline Outpatient & 0.558 & $0.3-0.8$ & {$[17]$} \\
\hline Hospitalized & 0.2 & $0.1-0.4$ & Estimate \\
\hline \multicolumn{4}{|l|}{ Pertussis } \\
\hline Mild & 0.9 & $0.8-0.99$ & [14] \\
\hline Moderate & 0.85 & $0.75-0.95$ & [14] \\
\hline Severe & 0.81 & $0.6-0.9$ & [14] \\
\hline Encephalopathy & 0.2 & $0-0.4$ & [14] \\
\hline \multicolumn{4}{|l|}{ Non-bacteremic pneumococcal pneumonia } \\
\hline Inpatient & 0.2 & $0-0.5$ & Estimate [32] \\
\hline Outpatient & 0.9 & $0.7-1$ & Estimate \\
\hline Invasive pneumococcal disease & 0.2 & $0-0.5$ & [32] \\
\hline Disability post pneumococcal disease & 0.4 & $0.2-0.6$ & Estimate [33] \\
\hline Disutilities (quality adjusted life years lost) & QALY & QALY & \\
\hline Illness death (discounted) & 10.25 & $5-15$ & [18] \\
\hline
\end{tabular}


Table 1 Model parameter values for high-risk adults aged 18-64 years (Continued)

\begin{tabular}{|c|c|c|c|}
\hline Parameter & Base case & Range & Source \\
\hline \multicolumn{4}{|l|}{$\begin{array}{l}\text { Durations (days lost due to illness) } \\
\text { Illness duration (days) }\end{array}$} \\
\hline Influenza & Days & Days & \\
\hline Outpatient & 4 & $1-8$ & [12] \\
\hline Hospitalized & 24 & $15-35$ & [12] \\
\hline Pertussis & 87 & 68-107 & [14] \\
\hline \multicolumn{4}{|c|}{ Non-bacteremic pneumococcal pneumonia } \\
\hline Inpatient & 27 & $18-38$ & [34] \\
\hline Outpatient & 18 & $11-26$ & [34] \\
\hline Invasive pneumococcal disease & 27 & $18-38$ & [34] \\
\hline
\end{tabular}

Tdap Tetanus, diphtheria, pertussis vaccine, IPD Invasive pneumococcal disease, NBP Non-bacteremic pneumococcal pneumonia, PCV13 13-valent pneumococcal conjugate vaccine, PPSV Pneumococcal polysaccharide vaccine

${ }^{a}$ Versus vaccine serotype

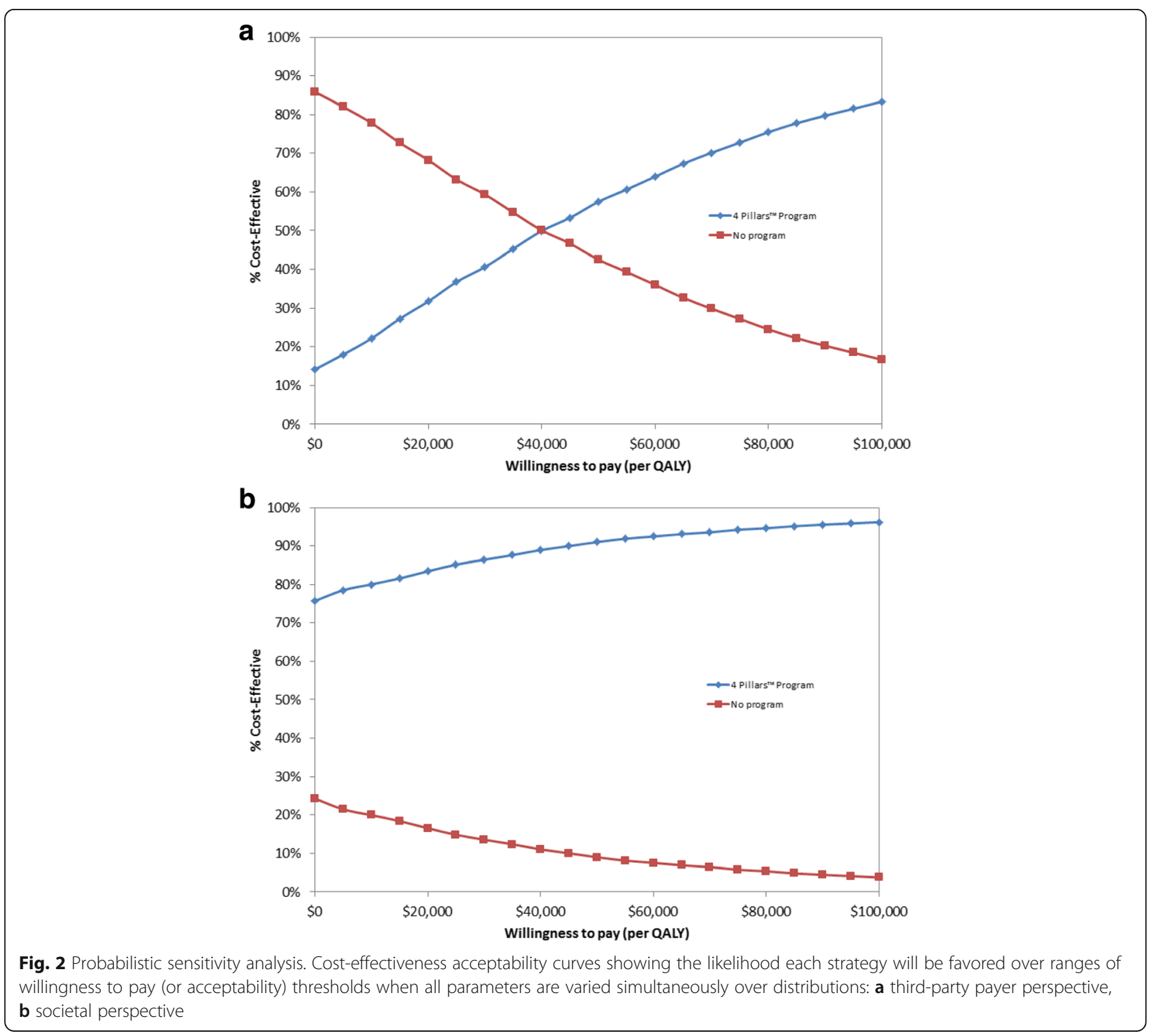


Table 2 Public health outcome predictions - vaccination programs in high-risk adults aged 18-64 years

\begin{tabular}{lll}
\hline & \multicolumn{2}{l}{ Strategy (cases per 100,000) } \\
\cline { 2 - 3 } I Pillars Program & No Program \\
\hline Cases & 32,898 & 34,270 \\
Hospitalizations & 679 & 707 \\
Deaths & 44 & 46 \\
Pertussis & & \\
Cases & 1759 & 1815 \\
Severe Cases & 53 & 54 \\
Deaths & 0.454 & 0.468 \\
Pneumococcal Disease & & \\
IPD cases & 162 & 168 \\
IPD deaths & 26 & 27 \\
NBP hospitalized & 1898 & 1926 \\
NBP outpatient & 17,226 & 17,479 \\
NBP deaths & 119 & 121 \\
\hline IPD Invasive pneumococcal disease, NBP Nonbacteremic pneumococcal pneumonia
\end{tabular}

From a societal perspective, adding lost productivity costs, the 4 Pillars Program had per-person total costs that were $\$ 31.15$ less than no program while remaining more effective, thus dominating the no program strategy.

When varying each parameter individually in 1-way sensitivity analysis over the ranges shown in Table 1 , the intervention program remained favored from both third party and societal perspectives with each individual parameter variation when using a $\$ 100,000 / Q A L Y$ threshold, a commonly cited U.S. benchmark [20]. If the program was completely ineffective at increasing influenza vaccination rates or if influenza vaccine effectiveness was $20 \%$ yearly throughout the 10-year time horizon (base case 59\%), then the program cost about $\$ 75,000$ per QALY gained from a third-party payer perspective; these 2 parameters, both related to influenza vaccination, were the most sensitive to variation. With individual variation of all other parameters over clinically plausible ranges as listed in Table 1, model results were robust, with the intervention strategy remaining favored throughout.

Probabilistic sensitivity analysis results, where all parameters were simultaneously varied over distributions, are depicted in Fig. 2 as acceptability curves. At a $\$ 100,000 /$ QALY gained threshold, the 4 Pillars Program was favored in $83.3 \%$ of the model iterations from a third party perspective and in $96.2 \%$ of model iterations from a societal perspective. From a societal perspective, the program was cost saving and more effective than no program in $75.7 \%$ of model iterations.

\section{Discussion}

Our analysis suggests that implementing an intervention program, such as the 4 Pillars Program, to increase vaccination uptake prevented more illness and was likely economically favorable in an 18-64 year old adult population with immunocompromising conditions or other comorbid conditions that confer a high risk of vaccinepreventable disease. From a third-party payer perspective considering direct medical costs, the incremental cost-effectiveness ratio of \$28,301/QALY gained with program use was well within the benchmark $\$ 100,000 /$ QALY gained threshold for economic favorability; [20] from a societal perspective that additionally considered lost productivity costs, the intervention program was less costly and more effective than no intervention; i.e., a potentially cost-saving intervention that improves health outcomes. Results were robust in sensitivity analyses, with no individual parameter variation causing the intervention to cost more than $\$ 100,000$ per QALY gained and simultaneous variation of all parameters showing that the intervention was highly likely to be favored over no intervention.

Currently, the proportion of adults with high-risk conditions in the U.S. ranges from $12.4 \%$ in the $19-24$ yearold age group to $30.6 \%$ in persons $50-64$ years of age [1]. These proportions are expected to grow as obesity rates continue to climb, with their attendant increases in diabetes, cardiovascular disease, and other obesityrelated diseases. A practice-based intervention, such as the 4 Pillars Practice Transformation Program, is an

Table 3 Cost-effectiveness analysis of 4 Pillars Transformation Program in high-risk adults aged 18-64 years

\begin{tabular}{|c|c|c|c|c|c|}
\hline Strategy & Cost per person & Incremental Cost & Effectiveness (QALY) & Incremental Effectiveness (QALY) & ICER $^{\mathrm{a}}(\$ / \mathrm{QALY}$ \\
\hline \multicolumn{6}{|c|}{ 3rd Party Payer Perspective } \\
\hline No Program & $\$ 1624.44$ & - & -0.02808 & & \\
\hline 4 Pillars & $\$ 1642.32$ & $\$ 17.88$ & -0.02744 & 0.00063 & $\$ 28,301$ \\
\hline \multicolumn{6}{|c|}{ Societal Perspective } \\
\hline 4 Pillars & $\$ 3781.77$ & - & -0.02744 & & \\
\hline No Program & $\$ 3812.92$ & $\$ 31.15$ & -0.02808 & -0.00063 & Dominated $^{\mathrm{b}}$ \\
\hline
\end{tabular}

aCER = Incremental Cost-Effectiveness Ratio

${ }^{\mathrm{b}}$ Costs more and less effective when compared to alternate strategy 
excellent method to reach high-risk groups, because their chronic conditions typically increase the frequency with which they receive medical care. The modeled public health impact of the intervention program is notable, with substantial reductions in influenza cases and hospitalizations and in pneumococcal illness. This combination of public health benefit and economical reasonableness suggest that quality improvement efforts to increase high-risk adult vaccinations using the 4 Pillars Program are a worthwhile investment.

Many cost-effectiveness analyses of vaccine strategies can be found in the literature, mostly highly favorable toward vaccination, but fewer consider the costeffectiveness of intervention programs designed to improve vaccination rates. Analyses examining the costeffectiveness of vaccine uptake intervention programs mainly focused on adults aged 65 and older [9, 21-23]. One study compared the same 4 Pillars intervention in this analysis while the others examined different hypothetical programs of varying intensity in an elderly, minority population. These decision analysis-based studies found an increase in incremental effectiveness and slightly higher incremental costs when compared to no intervention, resulting in ICERs well below a \$50,000/QALY gained threshold, indicating economic favorability.

\section{Strengths and limitations}

This study is based on clinical trial results and incorporates both third-party and societal perspectives per recent guidelines [19]. All decision analyses and their results are subject to the parameters selected, although results were robust in 1-way and probabilistic sensitivity analyses. Influenza vaccination uptake and effectiveness were most sensitive to variation; thus, factors that interfere with program-related improvements in influenza vaccine uptake or diminish influenza vaccine effectiveness could impact the cost-effectiveness of the intervention. However, model results were not substantially affected by individual variation of all other parameter values, with the intervention remaining the favored strategy with individual and collective parameter variation. Another limitation is that we modeled the probability of receiving one vaccine as independent of receipt of other vaccines, in keeping with an overall public health impact perspective. In addition, we cannot separate the impact of each of the 4 Pillars. While the 4 Pillars program does allow practices to implement only those components of the program that each practice feels will be implementable and successful, studies have shown that implementation of only one or a relative few of the those components will not lead to success [24-26]. Finally, we assume that the pneumococcal polysaccharide vaccine is ineffective in preventing pneumococcal non-bacteremic pneumonia, which could be a controversial contention
[27]. However, if this vaccine is effective against NBP, the intervention becomes even more favorable compared to no program.

\section{Conclusion}

The 4 Pillars Practice Transformation Program is a costeffective or cost-saving strategy, depending on the perspective taken, for averting vaccine preventable diseases in adults aged less than 65 years with medical conditions that place them at higher risk for influenza complications.

\section{Additional file}

Additional file 1: Table S1. Description of data: Summary of intervention cost by center. (DOCX $17 \mathrm{~kb}$ )

\section{Abbreviations}

CDC: Centers for Disease Control and Prevention; HIV: human immunodeficiency virus; ICER: Incremental cost effectiveness ratio; IPD: Invasive pneumococcal disease; NBP: Non-bacteremic pneumococcal pneumonia; PCV13: 13-valent pneumococcal conjugate vaccine; PPSV: 23-valent pneumococcal polysaccharide vaccine; QALY: Quality- adjusted life year; Tdap: Tetanus-diphtheria-acellular pertussis vaccine; U.S.: United States

\section{Acknowledgements}

Not applicable.

\section{Funding}

Supported by a grant (U01 IP000662) from the Centers for Disease Control and Prevention. The views expressed herein are those of those authors and not those of the Centers for Disease Control and Prevention. Also supported by the National Institutes of Health (UL1 RR024153 and UL1TR000005), and by the National Institute of Allergy and Infectious Diseases (R01 Al116575).

\section{Availability of data and materials}

The datasets used and/or analyzed during the current study are available from the corresponding author on reasonable request

\section{Authors' contributions}

ARW and KJS performed the analysis, based on data from a study by RKZ, MPN, and CJL. ARW and KJS drafted the manuscript with critical revision by RKZ, MPN, and CJL. All authors read and approved the final manuscript.

Ethics approval and consent to participate

Most data used in the model came from publically available and/or published data; use of this data is exempt from ethics review in the U.S. https://www.hhs.gov/ohrp/regulations-and-policy/regulations/45-cfr-46/ index.html\#46.101. However some data came from the 4 Pillars cluster randomized trial. In that study, standard informed consent procedures were followed, and trial protocols were approved by institutional review boards of the University of Pittsburgh, Baylor College of Medicine, and the Harris Health System.

\section{Consent for publication}

Not applicable.

\section{Competing interests}

Dr. Zimmerman and Dr. Lin have active research grants from Sanofi Pasteur, Merck \& Co., Inc., and Pfizer Inc. Dr. Nowalk has received or currently receives grant funding from Merck \& Co., Inc., Pfizer, Inc., and Medlmmune, LLC. All other authors have no competing interests to disclose.

\section{Publisher's Note}

Springer Nature remains neutral with regard to jurisdictional claims in published maps and institutional affiliations. 


\section{Author details}

'Department of Medicine, University of Pittsburgh School of Medicine, 200 Meyran Ave., Suite 200, Pittsburgh, PA 15213, USA. ²Department of Family Medicine, University of Pittsburgh School of Medicine, Pittsburgh, PA, USA.

\section{Received: 10 March 2017 Accepted: 17 January 2018}

Published online: 25 January 2018

\section{References}

1. Zimmerman RK, Lauderdale DS, Tan SM, Wagener DK. Prevalence of highrisk indications for influenza vaccine varies by age, race, and income. Vaccine. 2010;28(39):6470-7.

2. Centers for Disease Contol and Prevention. Adult conditions immunization schedule. National Center for Immunization and Respiratory Diseases. 2016; http://www.cdc.gov/vaccines/schedules/hcp/imz/adult-conditions.html. Accesssed 15 June 2016

3. Williams WW, Lu P-J, O'Halloran A, et al. Surveillance of vaccination coverage among adult populations- United States, 2014. MMWR Surveill Summ. 2016;65(1):1-40.

4. Zimmerman RK, Brown AE, Pavlik VN, et al. Using the 4 pillars practice transformation program to increase pneumococcal immunizations for older adults: a cluster-randomized trial. J Am Geriatr Soc. 2017:65(1):114-22.

5. Lin CJ, Nowalk MP, Pavlik VN, et al. Using the 4 pillars ${ }^{\mathrm{TM}}$ practice transformation program to increase adult influenza vaccination and reduce missed opportunities in a randomized cluster trial. BMC Inf Dis. 2016;16(1):623.

6. Nowalk MP, Lin CJ, Pavlik VN, et al. Using the 4 pillars ${ }^{T M}$ practice transformation program to increase adult Tdap immunization in a randomized controlled cluster trial. Vaccine. 2016:34(41):5026-33.

7. Nowalk MP, Bolan N, Nutini J, et al. Success of the 4 pillars toolkit for influenza and pneumococcal vaccination in adults. J Healthc Qual. 2014; 36(6):5-15.

8. Zimmerman RK, Nowalk MP, Lin CJ, et al. Cluster randomized trial of a toolkit and early vaccine delivery to improve childhood influenza vaccination rates in primary care. Vaccine. 2014;32(29):3656-63.

9. Smith KJ, Zimmerman RK, Nowalk MP, Lin CJ. Cost-effectiveness of the 4 pillars practice Transormation program to improve vaccination of adults aged 65 and older. J Am Geriat Soc. 2017;65(4):763-78.

10. Smith K, Wateska AR, Nowalk MP, Raymund M, Nuorti JP, Zimmerman RK. Cost-effectiveness of adult vaccination strategies using pneumococcal conjugate vaccine compared with pneumococcal polysaccharide vaccine. JAMA. 2012:307(8):804-12.

11. Bonten MJM, Huijts SM, Bolkenbaas M, et al. Polysaccharide conjugate vaccine against pneumococcal pneumonia in adults. New Engl J Med. 2015; 372:1114-25.

12. Molinari N-AM, Ortega-Sanchez IR, Messonnier ML, et al. The annual impact of seasonal influenza in the US: measuring disease burden and costs. Vaccine. 2007;25(27):5086-96.

13. Weycker D, Sato R, Strutton D, Edelsberg J, Atwood M, Jackson LA. Public health and economic impact of 13-valent pneumococcal conjugate vaccine in US adults aged $\geq 50$ years. Vaccine. 2012;30:5437-44.

14. McGarry L, Krishnarajah G, Hill G, et al. Cost-effectiveness of tdap vaccination of adults aged $\geq 65$ years in the prevention of pertussis in the US: a dynamic model of disease transmission. PLoS One. 2014;9(1):e72723.

15. Masseria C, Krishnarajah G. The estimated incidence of pertussis in people aged 50 years old in the United States, 2006-2010. BMC Inf Dis. 2015;15(1):534.

16. Moore MR, Link-Gelles R, Schaffner W, et al. Effect of use of 13-valent pneumococcal conjugate vaccine in children on invasive pneumococcal disease in children and adults in the USA: analysis of multisite, populationbased surveillance. Lancet Infect Dis. 2015;15:301-9.

17. Lee GM, LeBaron C, Murphy TV, Lett S, Schauer S, Lieu TA. Pertussis in adolescents and adults: should we vaccinate? Pediatrics. 2005;115(6):1675-84.

18. Arias E. United States life tables, 2011. Natl Vital Stat Rep. 2015;64(11):1-63.

19. Sanders GD, Neumann PJ, Basu A, et al. Recommendations for conduct, methodological practices, and reporting of cost-effectiveness analyses: second panel on cost-effectiveness in health and medicine. JAMA. 2016; 316(10):1093-103.

20. Neumann PJ, Cohen JT, Weinstein MC. Updating cost-effectiveness - the curious resilience of the $\$ 50,000-$ per-QALY threshold. N Engl J Med. 2014; 371(9):796-7.
21. Michaelidis Cl, Zimmerman RK, Nowalk MP, Smith KJ. Cost-effectiveness of programs to eliminate disparities in elderly vaccination rates in the United States. BMC Public Health. 2014;14:718.

22. Michaelidis $\mathrm{Cl}$, Zimmerman RK, Nowalk MP, Smith KJ. Cost-effectiveness of a program to eliminate disparities in pneumococcal vaccination rates in elderly minority populations: an exploratory analysis. Value Health. 2013; 16(2):311-7.

23. Michaelidis $\mathrm{Cl}$, Zimmerman RK, Nowalk MP, Smith KJ. Estimating the costeffectiveness of a national program to eliminate disparities in influenza vaccination rates among elderly minority groups. Vaccine. 2011;29(19):3525-30

24. Zimmerman RK, Moehling KK, Lin CJ, et al. Improving adolescent HPV vaccination in a randomized controlled cluster trial using the 4 pillars $^{\text {TM }}$ practice transformation program. Vaccine. 2017;35:109-17.

25. Hawk M, Nowalk MP, Moehling KK, et al. Using a mixed methods approach to examine practice characteristics associated with implementation of an adult immunization intervention using the 4 pillars practice transformation program. J Healthc Qual. 2017;39(3):153-67.

26. Nowalk MP, Zimmerman RK, Lin CJ, et al. Maintenance of increased childhood influenza vaccination rates 1 year after an intervention in primary care practices. Acad Pediatr. 2016;16(1):57-63.

27. Moberley S, Holden J, Tatham D, Andrews R. Vaccines for preventing pneumococcal infection in adults. Cochrane Database Syst Rev. 2013;1:CD000422.

28. Osterholm MT, Kelley NS, Sommer A, Belongia EA. Efficacy and effectiveness of influenza vaccines: a systematic review and meta-analysis. Lancet Infect Dis. 2012;12(1):36-44.

29. Koepke R, Eickhoff JC, Ayele RA, et al. Estimating the effectiveness of tetanus-diphtheria-acellular pertussis vaccine (tdap) for preventing pertussis: evidence of rapidly waning immunity and difference in effectiveness by tdap brand. J Infect Dis. 2014;210(6):942-53.

30. CDC Vaccine Price List. Centers for Disease Control and Prevention. http://www.cdc.gov/vaccines/programs/vfc/awardees/vaccinemanagement/price-list/. Accessed 26 May 2016.

31. Centers for Medicare \& Medicaid Services. Physician fee schedule search. www.cms.gov/apps/physician-fee-schedule/search/search-criteria.aspx Accessed 15 Oct 2015

32. Sisk JE, Whang W, Butler JC, Sneller V-P, Whitney CG. Cost-effectiveness of vaccination against invasive pneumococcal disease among people 50 through 64 years of age: role of comorbid conditions and race. Ann Intern Med. 2003;138(12):960-8.

33. Gold MR, Franks P, McCoy K, Fryback DG. Toward consistency in cost-utility analyses: using national measures to create condition-specific values. Med Care. 1998;36(6):778-92

34. Stoecker C, Kim L, Gierke R, Pilishvili T. Incremental cost-effectiveness of 13valent pneumococcal conjugate vaccine for adults age 50 years and older in the United States. J Gen Intern Med. 2016;31(8):901-8.

\section{Submit your next manuscript to BioMed Central and we will help you at every step:}

- We accept pre-submission inquiries

- Our selector tool helps you to find the most relevant journal

- We provide round the clock customer support

- Convenient online submission

- Thorough peer review

- Inclusion in PubMed and all major indexing services

- Maximum visibility for your research

Submit your manuscript at www.biomedcentral.com/submit 